\title{
Radiotherapy in the acute medical setting
}

\author{
Authors: Sean Brown, ${ }^{A}$ Peter Kirkbride ${ }^{B}$ and Ernie Marshall ${ }^{C}$
}

\begin{abstract}
Radiotherapy is an effective treatment modality and an essential tool in the management of cancer. As the incidence of malignant disease rises it is inevitable that physicians will increasingly encounter patients who have presented acutely and require radiotherapy or with a complication from irradiation. This paper explores the basic principles of radiotherapy tailored to the perspective of the acute medical physician and how to manage acute complications. We also discuss the role of radiotherapy in the acutely ill patient and define the need for radiotherapy pathways to ensure that patients receive treatment in a timely manner.
\end{abstract}

\section{Introduction}

Radiotherapy (RT) is an integral tool in the management of malignant disease. Of all patients diagnosed with cancer at least half will receive radiation at some point in the course of their illness and consequently, physicians will be confronted with a significant number of cancer patients following emergency presentation that requires or is caused by radiation therapy.

\section{Background: radiation biology}

Radiation damages the DNA of cancer cells; its ionising effect causes DNA breaks which lead to cell death and tumour shrinkage. ${ }^{1}$ RT encompasses a range of differing treatments tailored according to intent; curative, adjuvant, radical, palliative and prophylactic (Box 1).

$\mathrm{RT}$ utilises therapeutic radiation that is part of the electromagnetic spectrum and consists of high energy photons: $\mathrm{X}$-rays. External beam RT is delivered from an external source and directed to a specific anatomical region that requires treatment. The source, termed a linear accelerator, directs high-energy electrons to a tungsten target producing a photon beam, and is housed within in a specific treatment facility, most often in the setting of a regional cancer specialist hospital. In brachytherapy, the source is placed in or next to a tumour within the patient (for example, in cervical cancer) and

Authors: Aspecialist registrar, Clinical Oncology, Clatterbridge Cancer Centre, Bebington, UK; ${ }^{B}$ consultant clinical oncologist, Clatterbridge Cancer Centre, Bebington, UK; ${ }^{C}$ consultant medical oncologist, Clatterbridge Cancer Centre, Bebington, UK radiation is emitted from the nucleus of a radioactive isotope; such as radium-223.

\section{The radiotherapy pathway}

Radiation is administered in daily treatments or 'fractions' that are typically delivered over a 30-minute appointment. In palliation, therapy may be delivered as a single fraction, however, curative therapy in many radiosensitive malignancies may require much higher doses in the range of 66-70 Gy, administered in fractions of 1.8-2 Gy daily throughout a 6-8 week schedule. Normal healthy tissues exhibit a threshold tolerance to RT, beyond which they become damaged and contribute to side effects. This threshold is what limits the dose that can be safely delivered. Time between fractions allows for normal cell repair. It also allows for sensitisation of tumour cells that were previously radioresistant. As tumour shrinkage occurs there is improved oxygenation to the cancer. This enhances the effect of RT via free radical production.

Complications of RT are described as 'early' (during or shortly after treatment) or 'late' (months to years after treatment). Higher RT doses translate into more early toxicity, sometimes this is unavoidable in curative treatment. Late complications are largely due to fibrosis of the irradiated tissue, the risk of which can be reduced by the use of smaller daily fractions of 2 Gy or less. Palliative RT treatment is intended to alleviate symptoms and is not curative. In this patient cohort, the focus is on quality of life and hence the acute effects of radical treatment would not be acceptable. Therefore treatment is administered with

\section{Key points}

Liaise with acute oncology teams for all suspected radiationinduced emergency presentations

Avoid treatment delays in radical radiotherapy (RT) patients

Understand the indications for emergency RT

Consider palliative RT in the context of holistic care alongside specialist palliative care and acute oncology services

Develop responsive urgent RT pathways with your acute oncology team

KEYWORDS: Radiotherapy, emergency admission, radical, palliative, adjuvant, acute medicine 


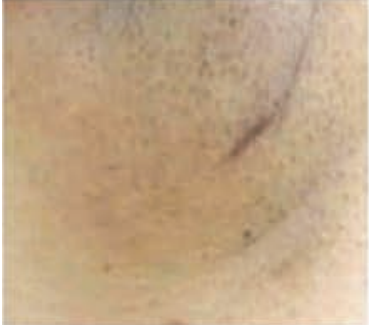

(a)

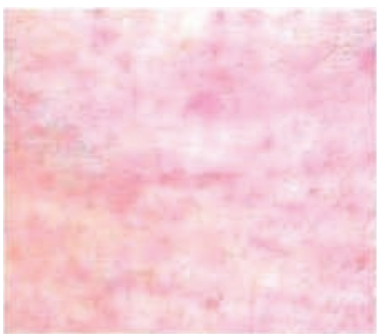

(e)

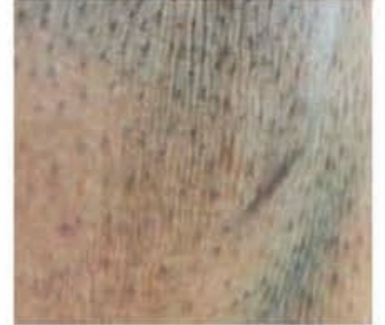

(b)

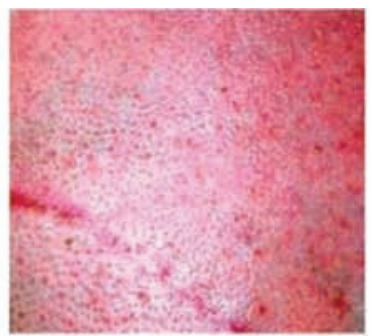

(f)

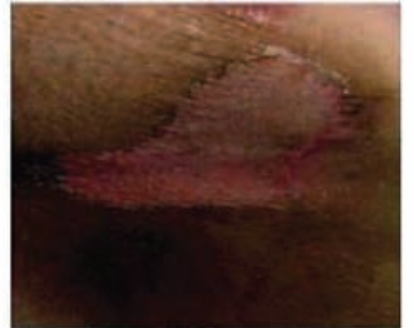

(c)

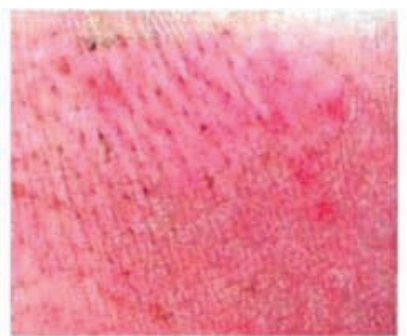

(g)

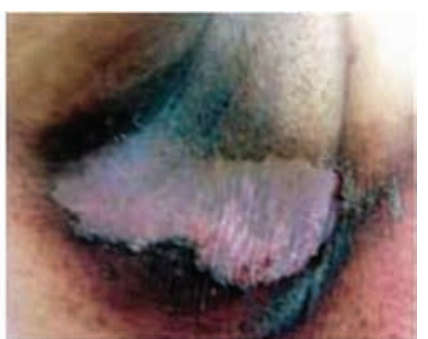

(d)

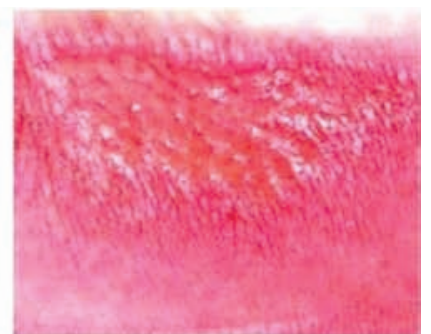

(h)

Fig 1. Stages of radiation dermatitis. Images show different stages of radiation dermatitis RTOG 1-4 in darkly-pigmented skin (a-d) and fair skin (e-h). Reproduced with permission. ${ }^{3}$ RTOG = Radiation Therapy Oncology Group.

fewer fractions of greater dose, given that late side effects, which occur over months to years, are less relevant to a population group with a shorter life expectancy.

\section{Side effects of radiotherapy}

RT is usually delivered from centralised regional centres; given this and the large volumes of patients treated; there will inevitably be occasions when patients will present to their local hospital with complications of their treatment. Therefore it is important for the medical team to be aware of the patterns of toxicity and general management principles.

The early complications of RT are common and usually selflimiting. They can peak after treatment has finished and cause anxiety if the patient is not informed of the nature and timing

\section{Box 1. Terms used to describe intention or RT.}

Curative: goal of treatment is elimination of tumour and cure of the patient.

Radical: refers to intensity of treatment and dose of radiation; high-dose treatment given to eradicate cancer from area treated.

Adjuvant: when RT is used in conjunction with primary therapy to aid the principal treatment regimen (eg radiation of the breast after a 'lumpectomy' to minimise the risk of local recurrence).

Palliative: treatment aimed at relieving or alleviating symptoms rather than cure.

Prophylactic: treatment given with preventative intent eg prophylactic cranial irradiation to prevent brain metastases by eliminating possible microscopic tumours in the brain, such as in small cell lung cancer.

$\mathrm{RT}$ = radiotherapy. of side effects before they occur. A common symptom is fatigue; the aetiology of which is not fully understood but can be seen in up to $80 \%$ of patients irradiated. ${ }^{2}$

As a general rule, RT acutely causes an inflammatory process in the area treated. This can present as swelling, oedema and pain. Once treatment finishes, inflammation and tissue irritation diminishes and these effects resolve. For example, RT to the head and neck typically causes painful ulceration of the oral mucosa (mucositis) as a consequence of radiation-induced stem cell loss within the epithelium. The gastrointestinal tract is equally sensitive and acute toxicity can manifest as oesophagitis from thoracic RT; nausea and vomiting from abdominal treatment. Fig 1 demonstrates the stages of radiation dermatitis.

The Radiation Therapy Oncology Group scoring criteria is commonly used to classify the severity of RT-induced side effects, the table below details the grades of side effects for common anatomical regions (Table 1$).{ }^{4}$

\section{Management of side effects}

Treatment for acute radiation toxicity is largely supportive and consists of analgesia, anti-inflammatory agents and measures to reduce the risk of secondary infection. Table 2 lists site-related side effects and specific treatments.

\section{Treatment interruptions}

RT is utilised in the treatment of $40 \%$ of patients who are cured of their cancer. However in order to maximise its efficacy it is essential that radiation is delivered within the timescale intended when initially prescribed. If treatment is interrupted or prolonged this may adversely affect patient outcome; an unscheduled gap of one day can result in an absolute reduction in local control of a tumour by $1.4 \%$ and therefore reduce the chance of cure. ${ }^{9}$ If a patient receiving RT is admitted to the 


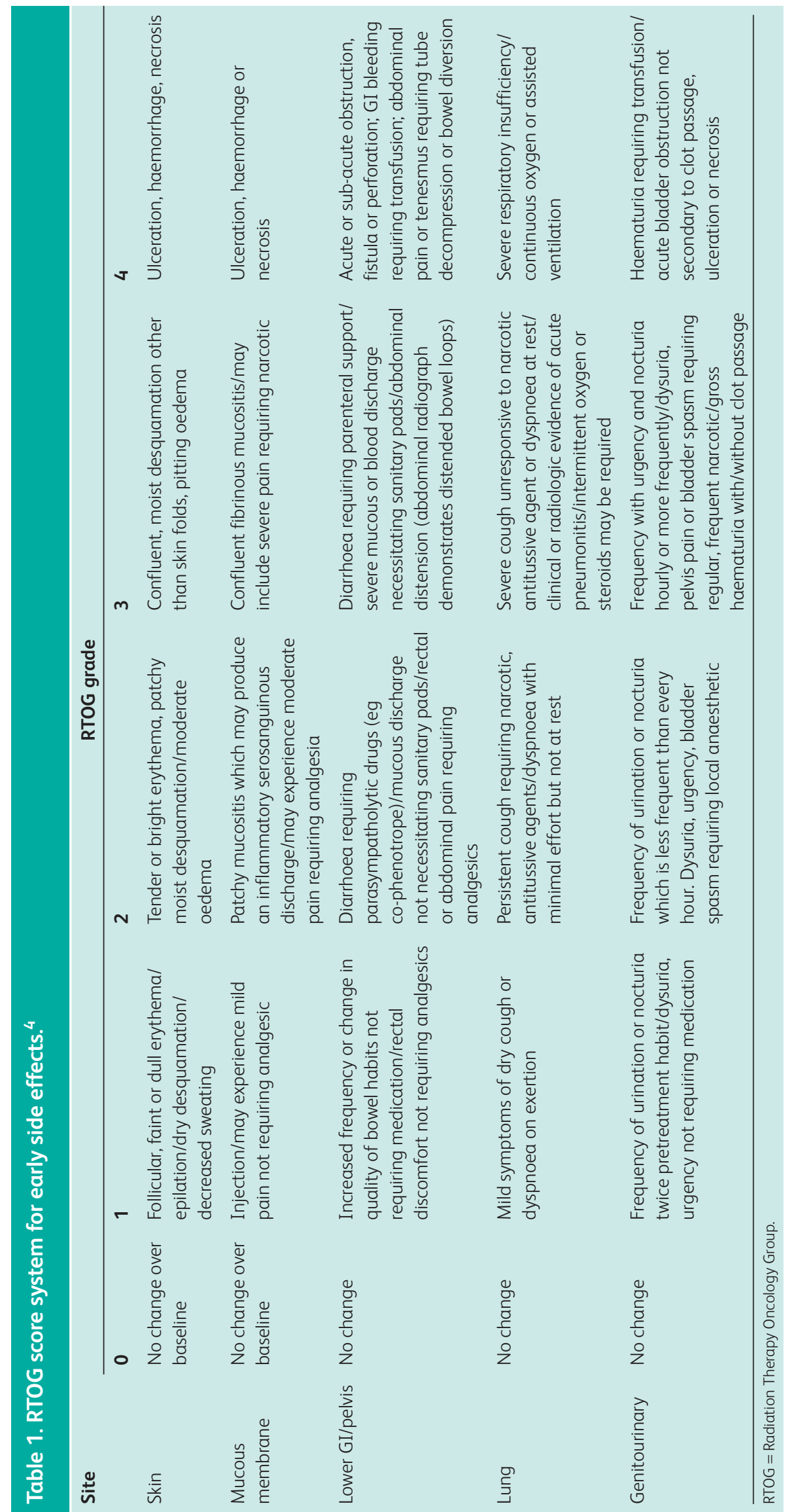




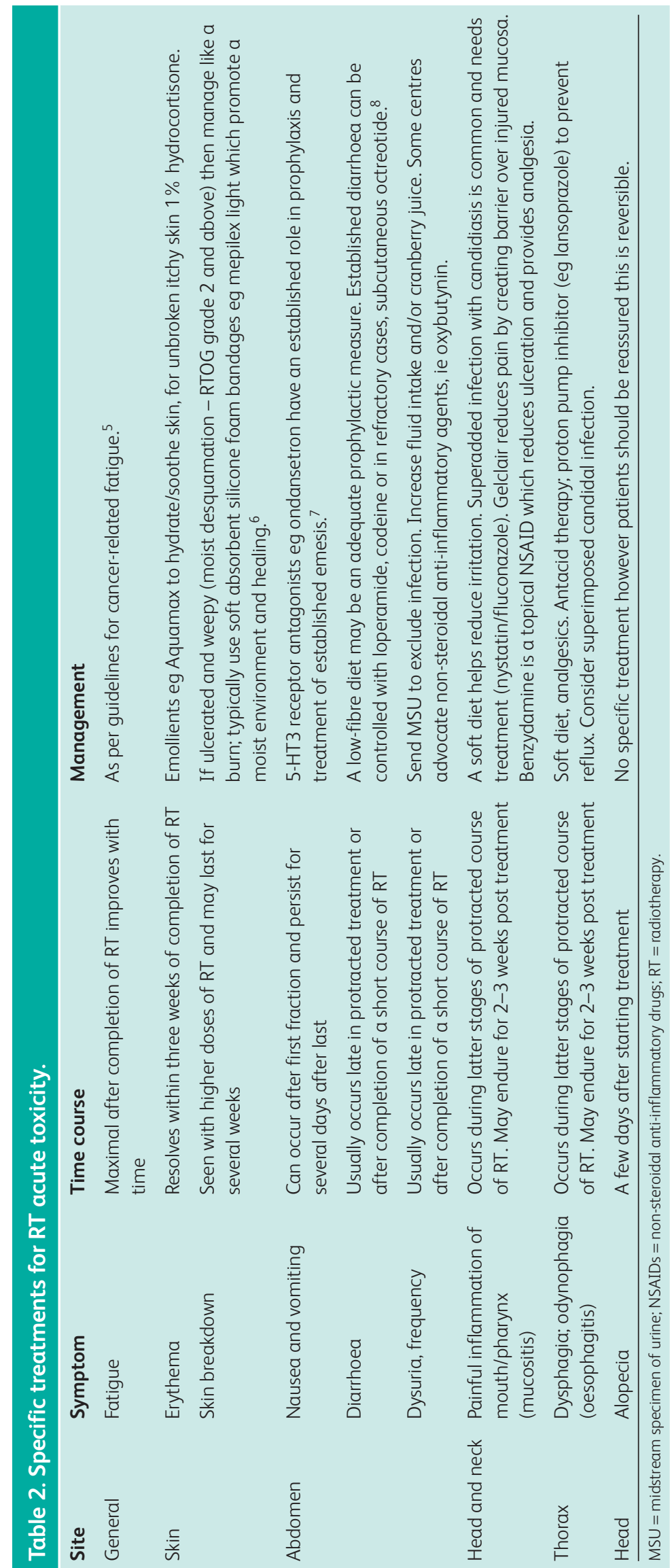




\section{Box 2. Indications for palliative RT.}

Pain relief (bone metastases, lung cancer causing chest pain, tumours causing nerve root and soft tissue infiltration)

Control of bleeding (haemoptysis, vaginal and rectal bleeding)

Control of fungation and ulceration

Relief of impending or actual obstruction (oesophagus, large airways or rectum)

Shrinkage of tumour masses causing symptoms (eg brain metastases or skin lesions)

Oncological emergencies (spinal cord compression or super mediastinal obstruction)

$\mathrm{RT}=$ radiotherapy.

medical assessment unit, the medical team must promptly liaise with the oncology services to determine if the admission is likely to cause a treatment gap, the implications of this and steps that can be taken to mitigate these.

\section{The role of radiotherapy in the acutely ill patient}

A significant number of cancer patients are admitted to hospital because of symptoms caused by their disease, rather than for complications of their treatment. RT is one of the most effective modalities of treatment for the relief of cancer symptoms such as pain and bleeding (see Box 2). It is estimated that approximately $40 \%$ of all courses of RT are given with palliative intent. ${ }^{10}$

Palliative RT can be used to relieve currently existing or incipient symptoms. In general it is best used for patients who have local symptoms that can be treated in the presence of a malignant tumour, although this can be extended to include patients who are deemed to be at risk of such symptoms.

\section{Common indications}

Pain control is the most common indication for palliative $\mathrm{RT}$ and should be considered in the context of a holistic care that includes appropriate analgesia and advice from specialist palliative care services. The holistic approach is particularly important, as maximum RT benefit may take several weeks to be achieved. Other potential indications for palliative RT are also included in Box 3.

In general, most palliative RT is given using comparatively low doses over as short a time as possible to minimise inconvenience to the patient. Single fractions are commonly used, but other typical regimes include two fractions given a week apart or five consecutive daily fractions.

The success rate of palliative RT depends on the indication for which it is being used, and it is sometimes difficult to measure, due to compounding factors such as a changing dose of analgesia. Nevertheless it is estimated that between 60 and $70 \%$ of patients who receive palliative RT for the treatment of symptoms caused by underlying malignancy do derive some benefit. ${ }^{11,12}$

\section{Contraindications}

There are actually few contraindications to palliative RT and it is important to liaise with acute oncology services in determining the role of RT for an individual patient. Many
Box 3. Radiation therapy emergency situations.

Established spinal cord compression

Established superior vena cava obstruction, if stenting not possible

Life-threatening lower airway obstruction

Life-threatening haemorrhage

Critical complications of radioresponsive tumours:

$>$ ocular compression with blindness

$>$ peripheral nerve compression, including cauda equina syndrome, with established motor dysfunction

> life-threatening renal insufficiency caused by kidney infiltration or ureteric obstruction

$>$ life-threatening mass brain lesion

$>$ Malignant hypercalcaemia refractory to other measures where RT constitutes appropriate definitive treatment

Progression of cancer documented following decision to treat with radiation therapy

$\mathrm{RT}=$ radiotherapy .

patients deemed not suitable for radical treatment on the grounds of poor performance status, co-existing medical conditions or disease extent, may benefit from palliative RT. For example, patients with poor lung function and limited respiratory reserve, unable to undergo surgery or radical RT, may benefit from low-dose RT with a lower risk of toxicity.

In terms of preventative RT there is little data to guide practice; for example it is not standard practice to prophylactically irradiate bone metastases whether or not they are painful, although there may be some benefits with the risk of fracture. However, lesions at high risk of fracture should preferentially be first reviewed by an orthopaedic surgeon to see if surgical intervention is more appropriate. There is no threshold of symptom severity that a patient needs to cross to require palliative RT. It is equally useful whether the disease is producing mild or severe symptoms, with little difference in palliative effect if treating a small or large volume of tumours.

\section{Urgent radiotherapy}

There are some situations when RT should be given urgently (ie within 24 or 48 hours), otherwise failure to do so could lead to death or catastrophic, irreversible damage. Severe pain, distressing as it may be to the patient, is therefore not an emergency, although it should be treated urgently. Box 3 is a list of RT emergency situations. Under these circumstances, ideally the patient should be seen by an oncologist and start treatment within 24 hours.

\section{Emergency radiotherapy pathways}

Pathways to access palliative RT for acute oncology patients can vary. In many centres, a patient who is admitted acutely unwell and found to have symptoms amenable to palliative RT, may have to be referred to a clinical oncologist, see that oncologist in clinic for a decision on RT treatment, and then wait to be planned for and to start treatment. However, other models 
exist that allow fast-tracking of acutely symptomatic patients to enable them to receive treatment in a timelier manner.

Although in many centres patients are booked into the next available planning slot for the relevant consultant, in others there is a more team-based approach. For example, a model pioneered in Toronto consisted of a dedicated team of radiation oncologists providing fast-track palliative RT clinics most days of the week, and who would accept referrals from oncological colleagues, outlying hospitals and sometimes GPs. Other centres employed a weekly 'bucket' clinic approach, whereby palliative RT patients who could not be accommodated by their consultant within a few days would be picked up in a weekly clinic, often done by the on-call oncologist, or as in the Sheffield model, in a teaching clinic for clinical oncology registrars.

It is recommended that the acute oncology teams work with their RT centre to develop timely and effective pathways that are appropriate for their local service. Ideally, according to the Joint Council for Clinical Oncology guidelines, patients receiving palliative $\mathrm{RT}$ should wait for no more than a maximum of 7 days, ${ }^{13}$ although this is not always achievable.

In many circumstances, a one-stop-shop approach is appropriate as there is evidence that in many situations single fractions of RT are as effective as prolonged courses. For example, the standard of care for patients with painful bone metastases is a single fraction of $8 \mathrm{~Gy}$, and so ideally, a patient can see the oncologist, have their treatment planned and receive it all with one visit to the cancer centre.

\section{Conclusions}

$\mathrm{RT}$ is an effective treatment for cancer. It has an established role in the curative setting and is a useful tool in the palliation of symptoms secondary to malignancy. Side effects of radiation are managed with supportive care and are usually self limiting. The acute oncology team are best placed to advise on this and should be involved at an early stage if a patient is admitted while undergoing RT. It is important to minimise any unnecessary delays in treatment which may compromise a potential curative outcome. There are well established pathways for conditions that require urgent RT (eg spinal cord compression), and for patients who require palliative RT on a less urgent basis (eg for pain), it is important that the acute oncology teams work with their RT centre to ensure a timely effective service is available.

\section{References}

1 Revell SH. Relationship between chromosome damage and cell death. In: Ishihara T, Sasaki MS (eds), Radiation-induced chromosome damage in man. New York, NY: AR Liss, 1983:113.

2 Jereczek-Fossa BA, Marsiglia HR, Orecchia R. Radiotherapy-related fatigue. Crit Rev Oncol Hematol 2002;41:317-25.

3 Ryan JL, Heckler CE, Ling M et al. Curcumin for radiation dermatitis: a randomised, double-blind, placebo-controlled clinical trial of thirty breast cancer patients. Radiat Res 2013;180:34.

4 Radiation Therapy Oncology Group. Acute radiation morbidity scoring criteria. Available online at http://rtog.org/ResearchAssociates/ AdverseEventReporting/AcuteRadiationMorbidityScoringCriteria. aspx [Accessed 1 June 2015].

5 National Comprehensive Cancer Network. Cancer related fatigue: clinical practice guidelines in oncology. Available online at www.nccn. org/professionals/physician_gls/pdf/fatigue.pdf [Accessed 1 June 2015].

6 Zhong WH, Tang QF, Hu LY, Feng HX. Mepilex Lite dressings for managing acute radiation dermatitis in nasopharyngeal carcinoma patients: a systematic controlled clinical trial. Med Oncol 2013;30:761.

7 Salvo N, Doble B, Khan L et al. Prophylaxis of radiation-induced nausea and vomiting using 5-hydroxytryptamine-3 serotonin receptor antagonists: a systematic review of randomized trials. Int J Radiat Oncol Biol Phys 2012;82:408.

8 Topkan E, Karaoglu A. Octreotide in the management of chemoradiotherapy-induced diarrhea refractory to loperamide in patients with rectal carcinoma. Oncology 2006;71:354.

9 Barton MB, Keane TJ, Galla T, Maki E. The effect of treatment time and treatment interruption on tumour control following radical radiotherapy of laryngeal cancer. Radiother Oncol 1992;24:137-43.

10 Kirkbride P, Barton R. Palliative radiation therapy. J Palliat Med 1999;2:87-97.

11 Reinfuss M, Mucha-Małecka A, Walasek T et al. Palliative thoracic radiotherapy in non-small cell lung cancer. An analysis of 1250 patients. Palliation of symptoms, tolerance and toxicity. Lung Cancer 2011 Mar;71:344-9.

12 Kirkbride P, Mackillop WJ, Priestman TJ et al. The role of palliative radiotherapy for bone metastases. Can J Oncol 1996 Feb;6 Suppl $1: 33-8$.

13 Joint Council for Clinical Oncology. Reducing delays in cancer treatment: some targets. joint collegiate council for oncology. London: Royal College of Physicians and The Royal College of Radiologists: 1993.

Address for correspondence: Dr S Brown, Clatterbridge Cancer Centre, Bebington, Wirral CH63 4JY, UK.

Email: sean.brown@clatterbridgecc.nhs.uk 